\title{
An integrated scheme to improve performance of fast mobile IPv6 handover in IEEE 802.16e network
}

\begin{abstract}
IEEE 802.16e standard for mobile Worldwide Interoperability for Microwave Access (WiMAX), defines the implementation of hard handover. The standard covers the physical and MAC layer. To apply the layer 3 (L3) handover protocol over the existing IEEE 802.16e layer 2 (L2) handover scheme, the fast mobile IPv6 was used by many researches, but long latency is main challenge for real-time applications. In this paper, to solve latency problem, an integrated scheme is proposed that it combines cross layer design and cross function optimization. The integrated scheme, bases on a pre-establish tunnels concept is dealt both, L2 handover in 802.16e and L3 handover in fast mobile IPv6 to effectively blend the relative messages of L2 and L3. The proposed scheme is compare with the scheme what standardized in IETF network working group (RFC5270). Analytic results prove that the proposed scheme can reduce not only handover latency but also packet losses.
\end{abstract}

Keyword: Fast mobile handover; Handover latency; IEEE802.16e; Packet loss; Pre-establish tunnel 